\title{
Clinical utility of QuantiFERON TB Gold Test in the diagnosis of latent tuberculosis infections
}

\author{
Salvatore Nisticò, Giuseppe Ivan Potente, Maria Teresa Cerminara, Lavinia Scuro, Angela Luciano \\ ASP Catanzaro, Presidio Ospedaliero di Lamezia Terme; S.C. Microbiologia e Virologia
}

Key words: tuberculosis, QuantiFERON-TB Gold, latent tuberculosis infection

Utilità clinica del QuantiFERON TB Gold Test nella diagnosi di infezione tubercolare latente

SUMMARY:

Introduction: Early diagnosis of latent tuberculosis infection can avoid a progression to active tuberculosis. The interferon gamma (IFN- $\gamma$ ) production measured by the new interferon-gamma release assays (IGRAs) can be used to identify subjects with, because the tuberculin skin test, is unreliable to discriminative between subjects with and others.

Methods: 186 patients tested positive with the tuberculin skin test were evaluated with QuantiFERON-TB Gold [QFT-G (Cellestis Ltd, Carnegie, Australia)].

Results: 6 I (32.8\%) samples showed a positive response with QFT-G test and only I (0.54\%) patient tested indeterminate.

Conclusion: These preliminary data suggest that QFT-G-positivity showed a strong correlation with clinical suspect of latent tuberculosis infection.

\section{INTRODUZIONE}

La tubercolosi (TB) attiva rappresenta ancora oggi una emergenza per la salute pubblica. Secondo le stime dell'Organizzazione Mondiale della Sanità (OMS) muoiono ancora oggi nel mondo circa 5000 malati al giorno e, anche se l'incidenza annuale della malattia è stabile dal 2005, la prevalenza continua ad aumentare. La situazione più allarmante si registra in Africa e nei Paesi dell'ex Unione Sovietica, sia per la scarsa efficienza dei sistemi di controllo della malattia sia a causa dell' emergenza di ceppi di micobatteri tubercolari resistenti ai farmaci di prima linea. L'identificazione ed il trattamento dei casi di TB attiva costituiscono una valida strategia per il controllo della malattia, tuttavia la trasmissione dell'infezione avviene in una fase precoce, prima ancora che venga diagnosticato il caso indice. L'infezione può evolvere, in base alla diversa risposta immunitaria dell'organismo, verso forme cliniche di malattia attiva severa o lieve oppure verso uno stato detto di infezione tubercolare latente (ITBL).

L'ITBL si caratterizza per l'assenza di una sintomatologia clinica manifesta e può portare , se non precocemente identificata, alla progressione verso la malattia attiva.

Il test cutaneo tubercolinico (TCT) mediante intrademoreazione secondo Mantoux rappresenta ancora oggi un ausilio diagnostico utilizzato per lo screening di soggetti a rischio di infezione latente (contatti del caso indice). Tuttavia è necessario tenere in considerazione alcuni importanti limiti del test TCT quali la bassa sensibilità e specificità, la variabilità di esecuzione e di lettura da parte del personale sanitario, la necessità di una visita di ritorno per la lettura del risultato e l'effetto "boosting” derivante da esecuzioni ripetute del test in tempi ravvicinati.

Dal 2005 è stato introdotto nella pratica clinica un test commerciale in vitro, il QuantiFERON-TB Gold (QFT-G) (Cellestis Ltd, Carnegie, Australia ), basato sul dosaggio dell'interferone-gamma (IFN- $\gamma$ ) prodotto dai linfociti T dopo stimolazione con antigeni specifici per il Mycobacterium tuberculosis (Mtb).

\section{MATERIALI E METODI}

Durante il biennio 2008-2009, 186 contatti di un caso indice di TB, positivi al TCT, con un quadro clinico/radiologico negativo per malattia attiva in atto sono stati sottoposti al test QFT-G.

Il test cutaneo tubercolinico (Biocine PPD liofilo, Novartis,
Siena, Italia) è stato eseguito inoculando per via intradermica $0.1 \mathrm{~mL}$ di tubercolina (PPD) contenente 5 unità, nella superficie volare dell'avambraccio. Il test è stato letto dopo 72 ore, valutando la reazione attraverso la palpazione dell'area eritematosa al fine di evidenziare la presenza dell'indurimento dell'infiltrato. L'intensità della reazione è stata determinata misurando il diametro traverso dell’infiltrato in $\mathrm{mm}$. Il test TCT è stato considerato debolmente positivo se l'indurimento in mm era compreso tra 6 e $10 \mathrm{~mm}$ e positivo se $\geq 10 \mathrm{~mm}$. Il QuantiFERON-TB Gold (QFT-G) (Cellestis Ltd, Carnegie, Australia) è un test che si basa sul rilascio e successivo dosaggio di IFN- $\gamma$ prodotto dai linfociti T attivati dopo stimolazione con antigeni Mtb-specifici, Early Secretory Antigen Target-6 (ESAT-6) e Culture Filtrate Protein-10 (CFP-10). Un'aliquota di $1 \mathrm{ml}$ di sangue intero eparinato dei pazienti in studio è stata raccolta in una provetta contenente gli antigeni Mtb-specifici, $1 \mathrm{ml}$ in provetta contenente una soluzione salina usata come controllo negativo per misurare i livelli basali di IFN- $\gamma$ ed un $1 \mathrm{ml}$ di sangue è stato stimolato con phytohaemoagglutinina (PHA) come controllo interno positivo.

Le provette sono state incubate a $37^{\circ} \mathrm{C}$ in atmosfera umidificata per $16-18$ ore. Dopo incubazione, i campioni sono stati centrifugati a 4000 rpm per 20 minuti. La concentrazione di IFN- $\gamma$ è stata misurata con sistema ELISA ed il livello di IFN- $\gamma$ rilasciato è stato calcolato sottraendo il livello di citochina misurato nel controllo negativo da quello misurato nel plasma dei campioni contenenti gli antigeni Mtb-specifici. Il risultato del test è stato calcolato utilizzando un software dedicato secondo le raccomandazioni fornite dal produttore. I livelli di IFN- $\gamma$ dosati sono stati espressi in Unità Internazionali/ml (UI/ml), con un cut-off di positività $\geq$ di $0.35 \mathrm{UI} / \mathrm{ml}$.

\section{RISULTATI E DISCUSSIONE}

Dei 186 campioni di plasma in esame, 61 (32.8\%) erano positivi al test QFT-G con livelli di IFN- $\gamma$ superiori al cut-off di $0.35 \mathrm{UI} / \mathrm{ml}$ ed uno solo (0.5\%) presentava un risultato indeterminato.

I dati preliminari dello studio hanno evidenziato una forte correlazione tra infezione tubercolare latente ed un risultato positivo con il test QuantiFERON-TB Gold. Questi risultati preliminari suggeriscono che il test QFT-G permette di identificare precocemente ed in maniera specifica i pazienti esposti con aumentato rischio di progressione da ITBL

\section{Corresponding author: Giuseppe Ivan Potente}

88100 Catanzaro - Via dei Normanni 117

Cell.: 3349457269

E-mail: ivanpotente@libero.it 
verso una TB attiva e che, pertanto, necessitano della somministrazione della terapia preventiva con isoniazide. L'evidenza clinica del nostro studio suggerisce che il test QFT-G, considerato i costi relativamente elevati rispetto al test tuberculonico, può essere utilizzato come test di screening su popolazioni di contatti come test di approfondimento diagnostico per quei pazienti che risultano positivi al test cutaneo tubercolinico e per i quali è consigliato il trattamento terapeutico con isoniazide al fine di evitare la progressione verso la malattia attiva.

\section{BIBLIOGRAFIA}

1. A.I.P.O. e S.I.Me.R. Documento di utilizzo dei nuovi test immunologici per la diagnosi di infezione tubercolare latente. Rass Patol App Respir 2007; 22: 13-20. Micobatteriologia clinica. Selecta Medica Pavia 2008.

2. A.T.S., C.D.C., Council of the Infectious Disease Society of America. Diagnostic standars and classification of tuberculosis in adult and children. Am J Resp Crit Care MED. 2000; 161: 1376-95.

3. C.D.C. Guidelines for using QuantiFERON-TB Gold test for detecting Mycobacterium tuberculosis infection. United States M.M.W.R. 2005; 54: 49-55.

4. W.H.O. Global truberculosis control. Surveillance, Planing, Financing. W.H.O. report 2007, W.H.O. Geneve. 